\title{
RAMAN SPECTRUM OF CRYSTALLINE AMINOSULPHONIC ACID
}

\author{
BY N. KRISHNAMURTHY \\ (Department of Physics, \\ Indian Institute of Science, Bangalore-12) \\ Received November 30, 1964 \\ (Communicated by Prof. R. S. Krishnan, I.A.sc.)

\section{INTRODUCTION}

AMINosulphonIC ACID or sulphamic acid $\mathrm{NH}_{3}+\mathrm{SO}_{3}-$ is the simplest derivative of sulphuric acid and has been known to be an interesting compound from the point of view of spectroscopic and structural studies (Vuagnant and Wagner, 1957; Gupta and Majumdar, 1941). At present, only an incomplete study of its Raman spectrum by Gupta and Majumdar (1941) is available. As large single crystals of sulphamic acid can be easily grown from aqueous solution, it was thought desirable to reinvestigate its Raman spectrum and to assess the nature and symmetry of these molecules in the crystal. The more detailed study of the infra-red absorption spectrum of sulphamic acid over a wide range of temperatures by Vuagnant and Wagner (1957) has been used in the assignment of the observed frequencies.

\section{Experimental Details}

Single crystals of sulphamic acid exhibiting well-defined faces could be easily grown from aqueous solutions of the pure substance. The biggest crystal grown had roughly the size $20 \times 10 \times 5 \mathrm{~mm}$. As the crystals were found to be transparent to the ultra-violet, the resonance radiation $\lambda 2537$ of mercury was used for the excitation of the Raman spectra. Preliminary experiments revealed the presence of very closely spaced lattice lines and therefore in the final intense spectra a fine slit of $0.015 \mathrm{~mm}$. was used. With the Hilger medium quartz spectrograph and Zenith Astronomical plates, exposures of about six hours were found to be necessary to record intense spectra.

\section{RESULTS}

Figure $1 b$ is an enlargement of the Raman spectrum of a single crystal of sulphamic acid with its microphotometer record in Fig. $1 c$. Figure $1 a$ 146 
is the comparison mercury spectrum in the ultra-violet region. The positions and frequency shifts of the Raman lines are marked on the microphotometer record. They are also listed in Table I. The figures given in brackets represent the relative Intensities of the Raman lines estimated visually. Thirty-eight distinct Raman lines have been recorded. Of these, the first seven belong to the lattice spectrum while the remaining ones arise from the internal oscillations. The infra-red data are also included in the same table. There is good agreement between the Raman and infra-red frequencies in the region investigated.

\section{Discussion}

Crystal structure data.-The crystal structure of sulphamic acid was determined by Kanda and King (1951) and by Osaki, Tadokoro and Nitta (1955) who found that sulphamic acid crystallises in the orthorhombic system with the space group $\mathrm{V}_{h}{ }^{15}-\mathrm{Pbca}$. The unit cell dimensions are $a=8 \cdot 115$ $\pm 0.001 \AA, b=8.066 \pm 0.001 \AA$ and $c=9.255 \pm 0.003 \AA$. There are eight molecules in the unit cell. The acid crystallises as Zwitter ion molecules $\mathrm{NH}_{3}+\mathrm{SO}_{3}{ }^{-}$of a distorted tetrahedral form with the existence of extensive hydrogen bonding of the type $\mathrm{N}^{+}-\mathrm{H}$.... O with each $\mathrm{NH}_{3}{ }^{+}$group entering into five bridging systems. The hydrogen bond distances vary from $2.82 \AA$ to $3.07 \AA$, which has been confirmed by neutron diffraction studies by Sass (1960). The five hydrogen bond distances are $2 \cdot 82 \AA, 2 \cdot 83 \AA, 2.94 \AA$, $3.02 \AA$ and $3.07 \AA$.

External oscillations.-The conventional group theoretical methods of Bhagavantam and Venkatarayudu (1948) can be applied to find the $(3 p-6)$ internal molecular modes each $q$ fold degenerate and $3 q$ lattice rocking and $(3 q-3)$ lattice translational modes of a unit cell with $q$, $p$-atomic molecules. Such a calculation was performed treating the Zwitter ion as a unit and showed that twelve rotatory type and twelve translatory type of lattice oscillations can be expected to appear in Raman effect. The discrepancy between the observed and the calculated number of lattice lines appears to be due to the overcrowding of all these lines in a region of small frequency shifts. On account of their high intensities, the lines at $110,120,140$ and $158 \mathrm{~cm} .^{-1}$ can be attributed to rotatory type of lattice oscillations. The lines at 170 and $240 \mathrm{~cm}^{-1}$ stand out clearly from the rest of the lattice lines in their breadth and structure even though their intensities are different. These two lines have been attributed to the vibrations of the hydrogen bonds $\mathrm{NH}-\mathrm{O}$. Similar low-frequency shifts due to hydrogen bond oscillations have been observed by Gross (1959) and Balasubramanian and Krishnan (1958). 
TABLE I

Vibration spectrum of sulphamic acid (frequency shifts in $\mathrm{cm}^{-1}$ )

$\begin{array}{lccc}\text { S1. No. } & \begin{array}{c}\text { Raman } \\ \text { effect } \\ \text { present }\end{array} & \begin{array}{c}\text { Infra-red } \\ \text { Vuagnant } \\ \text { and Wagner }\end{array} & \text { Assignment }\end{array}$

\begin{tabular}{|c|c|c|c|}
\hline 1 & $50(2)$ & .. & Lattice \\
\hline 2 & $68(1)$ & .. & $\eta$ \\
\hline 3 & $80(3)$ & .. & $"$ \\
\hline 4 & $110(3)$ & . & $"$ \\
\hline 5 & 125 ( 4) & .. & " \\
\hline 6 & 140 ( 4) & $\cdots$ & $"$ \\
\hline $\begin{array}{l}7 \\
8\end{array}$ & $\begin{array}{l}155(4) \\
170(2)\end{array}$ & $\ldots$ & NḦ.... O \\
\hline 9 & $240(5)$ & $\cdots$ & NH.... \\
\hline 10 & $357(10)\}$ & & \\
\hline 11 & $378(10)\}$ & 352 & $v_{11}(e)$ \\
\hline $\begin{array}{l}12 \\
13\end{array}$ & $\left.\begin{array}{l}535(10) \\
550(10)\end{array}\right\}$ & $\begin{array}{l}520 \\
540\end{array}$ & $\nu_{10}(e)$ \\
\hline 14 & $678(10)$ & 682 & $v_{s}\left(a_{1}\right)$ \\
\hline 15 & $697(10)$ & 695 & $\because$ \\
\hline 16 & $862(?)$ & $\cdots$ & . \\
\hline $\begin{array}{l}17 \\
18\end{array}$ & 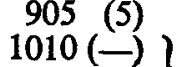 & $1 \overrightarrow{000}$ & (1) \\
\hline 19 & 1020 (8) & $1015\}$ & $\nu_{9}(e)$ \\
\hline 20 & $1057(20)$ & 1064 & $\nu_{k}\left(a_{1}\right)$ \\
\hline $\begin{array}{l}21 \\
22\end{array}$ & $\begin{array}{l}1080(10) \\
1265(10)\end{array}$ & 1262 & $\nu_{3}\left(a_{1}\right)$ \\
\hline 23 & $1280(10)$ & & $\therefore$ \\
\hline 24 & $1302(8)\}$ & 1312 & $v_{\mathrm{a}}(e)$ \\
\hline 26 & $\begin{array}{l}1319(10) \\
1440(6)\end{array}$ & $1 \ddot{446}$ & $\nu_{2}\left(a_{1}\right)$ \\
\hline $\begin{array}{l}27 \\
28\end{array}$ & $\left.\begin{array}{l}1534(5) \\
1571(8)\end{array}\right\}$ & $\left.\begin{array}{l}1542 \\
1570\end{array}\right\}$ & $\nu_{7}(e)$ \\
\hline & & $\begin{array}{l}1786 \\
2044 \\
2152 \\
2345\end{array}$ & \\
\hline 29 & $2460(5)$ & 2465 & $\nu_{2}\left(a_{1}\right)+\nu_{9}(e)^{*}$ \\
\hline 30 & $2476(4)$ & $\ldots$ & $\nu_{2}\left(a_{1}\right)+\nu_{3}(e)^{*}$ \\
\hline 31 & 2539 ( 3$)$ & $\cdots$ & $\nu_{7}(e)+\nu_{0}(e)^{-*}$ \\
\hline 32 & 2553 ( 2) & & $\nu_{7}(e)+\nu_{0}(e)^{*}$ \\
\hline 33 & $\begin{array}{l}2579(3) \\
2876(8)\end{array}$ & $\begin{array}{l}2569 \\
2893\end{array}$ & $\begin{array}{l}\nu_{7}(e)+\nu_{9}(e)^{*} \\
\mathrm{~N}-\mathrm{H} . \mathrm{O}_{\text {bonded }}\end{array}$ \\
\hline 35 & $\begin{array}{l}2876(8) \\
2906(2)\end{array}$ & & \\
\hline 36 & $3050(5)$ & 3055 & $\mathrm{~N}-\mathrm{H} . \ldots \mathrm{O}_{\text {bonded }}$ \\
\hline 37 & $3142(5)$ & 3140 & $\mathrm{~N}-\mathrm{H} . \ldots \mathrm{O}$ bonded \\
\hline 38 & $\begin{array}{l}3260 \text { to } \\
3450\end{array}$ & $\cdots$ & Band \\
\hline
\end{tabular}

- Combinations with the various components into which the degenerate mode is split up. 
Internal oscillations.-The free sulphamic acid molecule exists as a Zwitter ion having $\mathrm{C}_{3} v$ symmetry and hence its fundamental vibrations are distributed under the different symmetry types as below: $5 a_{1}+a_{2}+6 e$. The torsional vibration about the $\mathrm{N}-\mathrm{S}$ bond $\left(\nu_{12}\right)$ belonging to the $a_{2}$ type is forbidden to appear in the vibrational spectrum. All the remaining vibrations are active in both Raman effect and infra-red absorption. The vibrational assignments of the various modes of the free sulphamic acid are as follows:

$$
\begin{aligned}
& v_{1}\left(a_{1}\right) \text {-symmetric stretching } \mathrm{NH}_{3}^{+} \\
& v_{2}\left(a_{1}\right) \text {-symmetric deformation } \mathrm{NH}_{3}{ }^{+} \\
& v_{3}\left(a_{1}\right) \text {-symmetric stretching } \mathrm{SO}_{3}^{-} \\
& v_{4}\left(a_{1}\right) \text {-symmetric deformation } \mathrm{SO}_{3}^{-} \\
& v_{5}\left(a_{1}\right) \text {-N-S stretching } \\
& v_{6}(e) \text {-degenerate stretching } \mathrm{NH}_{3}^{+} \\
& v_{7}(e) \text {-degenerate deformation } \mathrm{NH}_{3}{ }^{+} \\
& v_{3}(e) \text {-degenerate stretching } \mathrm{SO}_{3}^{-} \\
& v_{9}(e) \text {-degenerate rocking } \mathrm{NH}_{3}{ }^{+} \\
& v_{10}(e) \text {-degenerate deformation } \mathrm{SO}_{3}^{-} \\
& v_{11}(e) \text {-degenerate rocking } \mathrm{SO}_{3}^{--} \\
& v_{12}\left(a_{2}\right) \text {-N-S torsion. }
\end{aligned}
$$

But in the crystal, the site symmetry of the Zwitter ion is $C_{1}$ and hence the degeneracies of the doubly degenerate modes are removed and also the torsional mode becomes active. Assuming that the site symmetry is not far from $\mathrm{C}_{s}$ due to the coupling of the modes of the eight molecules in the unit cell, one should have a total of one hundred and forty-four modes distributed among the various species as follows: $22 \mathrm{~A}_{1 g}+22 \mathrm{~B}_{1 g}+14 \mathrm{~B}_{2 g}+14 \mathrm{~B}_{3 g}$ $+14 A_{1 u}+14 B_{1 u}+22 B_{2 u}+22 B_{3 u}$ wherein the first four species are Raman active and the last three are infra-red active. The modes coming under $A_{1 u}$ are forbidden in both. Since the actual site symmetry is $C_{1}$ and there are eight molecules in the unit cell each mode coming under the species $A^{\prime}$ and $A^{\prime \prime}$ split into eight modes corresponding to all the eight species of the point group of the crystal and we arrive at the result that in the crystal one can expect 54 fundamental components in infra-red absorption and 72 in Raman effect.

In the observed Raman spectrum, we find that all the degenerate modes are split up (see Table I). The $\nu_{9}(e)$ mode falls near the mercury line at $\lambda 2603 \cdot 20 \AA$ and its appearance is verified by considering the relative intensity of the mercury line. The splitting of the degenerate modes varies from 
15 to $36 \mathrm{~cm} .^{-1}$ and indicates that the distortion of the Zwitter ion from the $\mathrm{C}_{3 v}$ symmetry is not very large. The coupling of the modes may give rise to elosely spaced lines and have therefore escaped detection due to the dispersion of the spectrograph.

An interesting feature of the observed spectrum is the high intensity of the $\nu_{4}\left(a_{1}\right)$ symmetric $\mathrm{SO}_{3}{ }^{-}$deformation vibration at $1057 \mathrm{~cm}^{-1}$ It is found to be excited even by the mercury lines $\lambda 2652.04 \AA, \lambda 2653.68 \AA$ and $\lambda 2655 \cdot 10 \AA$ and these are marked in the microphotometer record with shifts 2773,2796 and $2820 \mathrm{~cm}^{-1}$ The doubly degenerate $\mathrm{SO}_{3}$ deformation modes $\nu_{11}$ and $\nu_{10}$ appear with considerable intensity on the anti-Stokes side also. In the region of the $\mathrm{NH}_{3}{ }^{+}$stretching, there are four lines at 2876, 2908, 3050 and $3142 \mathrm{~cm}^{-1}$ The latter two are actually maxima in the band while the first is a very strong line. Its frequency shift is consistent with the reported $\mathrm{N}-\mathrm{H}$.... O hydrogen bond length of $2.82 \AA$ while the second may be the $\mathrm{N}-\mathrm{H}$. ... O bonded oscillation corresponding to the $\mathrm{N}-\mathrm{H}$.... O distance of $2.83 \AA$. The $3050 \mathrm{~cm}^{-1}$ line can be assigned to the hydrogen-bonded vibration for $\mathrm{N}-\mathrm{H}$.... O distance of $2.94 \AA$. These conclusions have been arrived at from the $\mathrm{N}-\mathrm{H}$ frequency and $\mathrm{N}-\mathrm{H}$... O distance curve reported by Krishnan and Krishnan (1964). The five broad and weak lines in the region of 2460-2579 cm.-1 cannot be explained as due to hydrogen bonds of lengths 2.82-3.07 $\AA$. and are therefore overtones and combinations of the fundamentals. The band extending from 3260 to $3450 \mathrm{~cm} .^{-1}$ has been assigned as due to the combinations involving the low frequency $\mathrm{NH}-\mathrm{O}$ vibrations and the $\mathrm{N}-\mathrm{H}$.... O hydrogen-bonded stretching vibrations. The absence of bands corresponding to $\mathrm{N}-\mathrm{H}$ stretching vibrations occurring beyond $3150 \mathrm{~cm} .^{-1}$ and the smaller splitting of the degenerate modes confirm the presence of Zwitter ions $\mathrm{NH}_{3}+\mathrm{SO}_{3}-$ and only the slight distortion of the molecular symmetry $C_{s v}$ of the free acid. It will be interesting to study the Raman spectra at low temperatures for understanding the related phenomena such as free rotation and the low-frequency hydrogen bond transitions.

\section{SUMMARY}

The Raman spectrum of a single crystal of sulphamic acid has been recorded with $\lambda 2537$ excitation. Thirty-eight lines have been observed, of which twenty-nine have been recorded for the first time. Seven Raman lines with shifts in the region $50-155 \mathrm{~cm}^{-1}$ have been assigned to the lattice oscillations, two at 177 and $240 \mathrm{~cm}^{-1}$ have been attributed to the low-frequency hydrogen bond vibrations. . The splitting of the degenerate modes and the appearance of $\mathrm{N}-\mathrm{H}$.... O bonded stretching vibrations are consistent with 
the structural data which expect the presence of the free molecule as a Zwitter ion with only slight distortion from $\mathrm{C}_{3 v}$ symmetry.

\section{ACKNOWLEDGEMENT}

The author wishes to express his heart-felt thanks to Prof. R. S. Krishnan for his suggestions and guidance.

\section{REFERENCES}

1. Bhagavantam, $S$. and Venkatarayudu, $\mathbf{T}$.

2. Gross, E. F. Theory of Groups and Its Applications to Physical Problems, Andhra University, Waltair, 1948.

. Hydrogen Bonding, by Hadzi, Pergamon Press, 1959, p. 203.

3. Gupta, S. J. and Majumdar, J. In. Chem. Soc., 1941, 18, 457. A. $K$.

4. Kanda, F. A. and King, A. J. Jour. Amer. Chem. Soc., 1957, 73, 2315.

5. Krishnan, R. S. and Bala- Proc. Ind. Acad. Sci., 1958, 47 A, 55; 1958, 48 A, 138. subramanian, $\mathbf{K}$.

6. __ and Krishnan, K. .. Ibid., 1964, 60 A, 11.

7. Osaki, K., Tadokoro, H. and Bull. Chem. Soc. (Japan), 1955, 28, 524. Nitta, I.

8. Sass, R. L. .. Acta Cryst., 1960, 13, 320.

9. Vuagnant, A. M. and Wagner, E. L.

J. Chent. Phys., 1957, 26, 77.

\section{Explanation of Plate XI}

Fig. 1. (a) Mercury spectrum.

(b) Raman spectrum of Sulphamic Acid.

(c) Microphotometer record. 


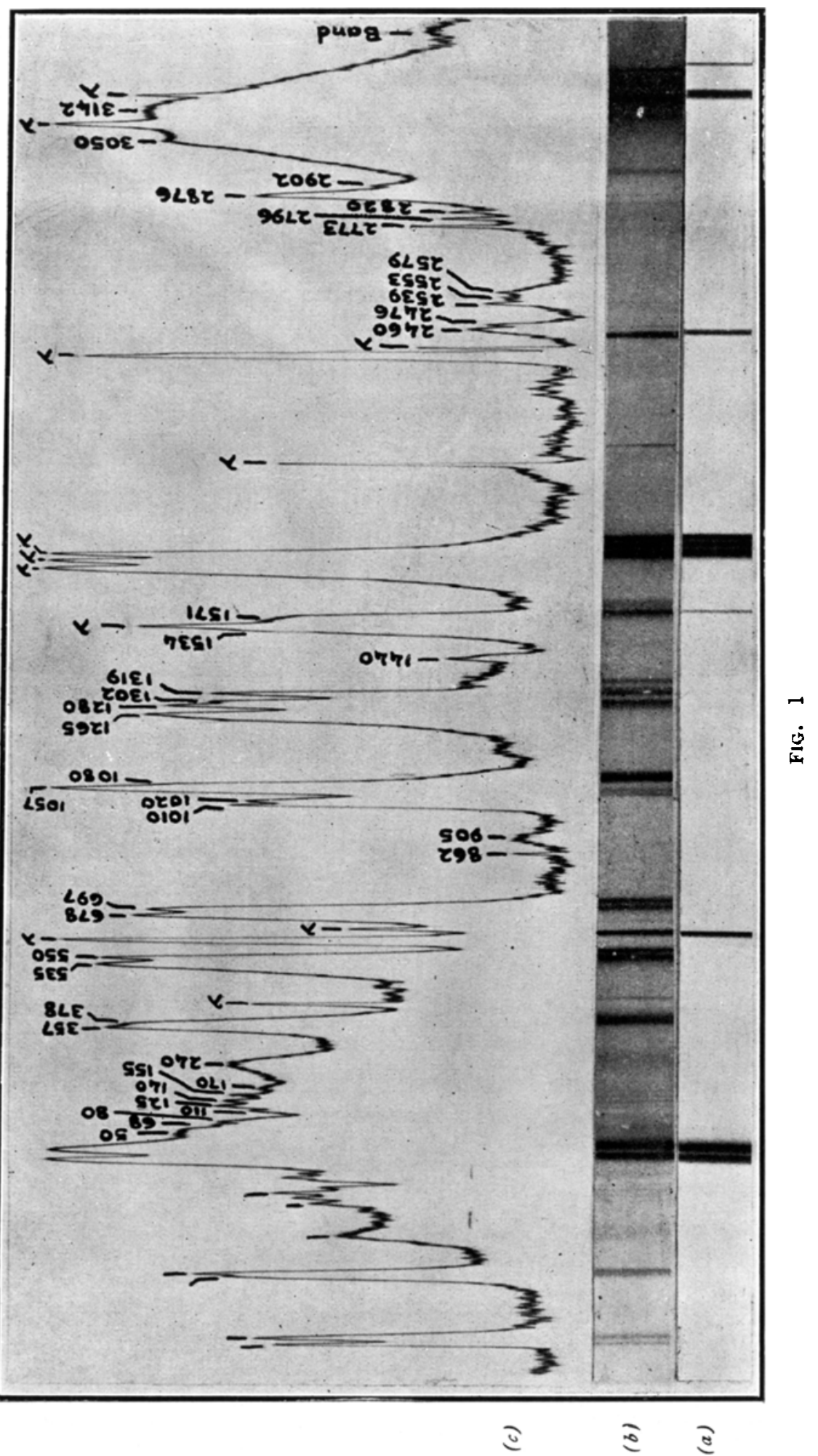

